\title{
GESTAÇÃO VIDA: OFICINA EDUCACIONAL PARA GESTANTES COM ABORDAGEM MULTIPROFISSIONAL EM UMA UNIDADE BÁSICA DE SAÚDE DA CIDADE DE MANAUS
}

\author{
Pregnancy life: educational workshop for pregnant women with multidisciplinary \\ approach in a basic health unit of the city of Manaus
}

\author{
Janaina Costa Lima ${ }^{1}$ \\ Danielle Bezerra Maia² \\ Paula Chagas de Farias Rocha ${ }^{3}$ \\ Rosângela Martins Gama ${ }^{4}$ \\ Regismeire Viana Lima ${ }^{5}$
}

\begin{abstract}
RESUMO
Este trabalho apresenta o relato de experiência dos residentes da Residência Multiprofissional em Saúde do Hospital Universitário Getúlio Vargas (HUGV), que através da atuação na atenção primária, pode contribuir com a promoção da saúde na comunidade do Armando Mendes, através de um projeto de extensão intitulado "Gestação vida: educando mamãe e bebê", com ações educativas voltadas para gestantes atendidas no pré-natal na Unidade de Saúde Básica (UBS) Geraldo Magela, na zona Leste de Manaus. O seu principal objetivo é relatar a experiência de uma abordagem multiprofissional na educação em saúde, através de uma oficina educativa para gestantes, sensibilizando-as quanto à importância de um pré-natal e de esclarecimento dos aspectos clínicos, psicológicos e sociais da gestação, bem como os cuidados necessários com os recém-nascidos. Conclui-se que a experiência como residentes na atenção básica nos mostrou a possibilidade da experimentação entre os saberes, contribuindo para a busca além do individual através de um modelo assistencial fundamentado na multiprofissionalidade, interdisciplinaridade e na humanização, considerando a integralidade do cuidado, demonstrando que é possível a ampliação do trabalho voltado para a complexidade do processo gravídico e puerperal.
\end{abstract}

Palavras-chave: multiprofissional; oficina; gestantes; pré-natal.

\footnotetext{
1 Graduada em Enfermagem pela Universidade Federal do Amazonas e Especialização em Enfermagem Intensiva de Alta Complexidade - UTI modalidade Adulto pela UniCel Faculdade Literatus (2010). Professora da Uninorte International Universities. E-mail: janaclima@hotmail.com 2 Bacharel em Serviço Social pela Universidade Federal do Amazonas. Especialização na modalidade de Residência Multiprofissional em Saúde. Mestranda do programa de mestrado de Serviço Social e Sustentabilidade na Amazônia da Universidade Federal do Amazonas. Professora da Escola Superior Batista do Amazonas. E-mail: daniellebmaia@hotmail.com

${ }^{3}$ Graduada em Farmácia pela Universidade Estácio de Sá, Especialista em Farmácia Hospitalar pela Sociedade Brasileira de Farmácia Hospitalar e Serviços de Saúde - SBRAFH. E-mail: paula_fc1981@hotmail.com

${ }^{4}$ Possui graduação em Educação Física pela Universidade Federal da Amazônia e mestrado em Actividade Física Adaptada pela Universidade do Porto. E-mail: rasagamartins@hotmail.com

${ }^{5}$ Mestrado Interinstitucional em Saúde Pública na Amazônia. Doutoranda em Saúde Pública na Universidade de São Paulo. E-mail: meirevi@hotmail.com
}

LIMA, Janaina C. et al. Gestação Vida: oficina educacional para gestantes com abordagem multiprofissional em uma Unidade Básica de Saúde da cidade de Manaus. Extensão em Foco, Curitiba: Editora da UFPR, nr.10, jul/dez 2014, p.86-101. ISSN 2358-7180. 


\begin{abstract}
This work presents an experience report of residents of the Multidisciplinary Residency at the Getúlio Vargas University Hospital (HUGV), which through the activities in primary care, may contribute to the promotion of health in the Armando Mendes community by means of an extension project titled "Pregnancy life: educating mom and baby" with educational actions focusing pregnant women attending prenatal care at Geraldo Magela Basic Health Unit (UBS) in the East zone of Manaus. Its main goal was reporting the experience of a multidisciplinary approach in health education through an educational workshop for pregnant women, sensitizing them on the importance of prenatal care and clarification of clinical, psychological and social aspects of pregnancy, as well as the necessary care to the newborns. It is concluded that the experience as residents in primary care showed us the possibility of experimentation among different kinds of knowledge, contributing to the search beyond the individual care through a model based on multidisciplinarity, interdisciplinarity and humanized actions, considering the comprehensiveness of the care and demonstrating that is possible to expand the work facing the complexity of pregnancy and the puerperal process.
\end{abstract}

Key words: multidisciplinary; workshop; pregnant women; prenatal care.

\title{
Introdução
}

O período da gravidez é marcado por intensas modificações fisiológicas, sociais e também psicológicas. Internamente, todo o seu organismo se prepara para abrigar um novo ser em desenvolvimento. Socialmente, a mulher se depara com toda a situação nova que terá que conviver, com um novo papel, o de ser mãe, alterando toda estrutura familiar que muda com a chegada do novo integrante (CAMACHO et al., 2010, p. 115-125).

O pré-natal torna-se de extrema importância para que a mãe e o bebê tenham uma assistência à saúde de forma integral. Porém, nem sempre se consegue suprir às gestantes de informações e cuidados suficientes para uma gestação mais tranquila, principalmente, levando em conta os aspectos sociais dessas usuárias, tanto no aspecto de informações, ações educativas e preventivas, como nos aspectos emocionais e sociais, sendo necessárias estratégias que ajudem a suprir essa carência de informação.

A gestação pode ser um momento propício para ações de promoção e educação em saúde, pois a mulher se mostra receptiva às mudanças e ao processamento de informações que possam ser revertidas em benefício ao bebê. Esta tem o papel-chave dentro da família, zelando pela sua saúde e a dos demais familiares, tornando-se multiplicadora de informações e ações que possam levar ao bem-estar do núcleo familiar e, consequentemente, a uma vida com qualidade. $\mathrm{A}$

LIMA, Janaina C. et al. Gestação Vida: oficina educacional para gestantes com abordagem multiprofissional em uma Unidade Básica de Saúde da cidade de Manaus. Extensão em Foco, Curitiba: Editora da UFPR, nr.10, jul/dez 2014, p.86-101. ISSN 2358-7180. 
aquisição de hábitos e escolhas saudáveis implica diretamente na mudança de comportamento, levando à promoção e manutenção de saúde do indivíduo (REIS et al., 2010, p. 269-276).

A Organização Mundial da Saúde (OMS) propõe recomendações essenciais para a atenção pré-natal e perinatal. Alguns dos dez princípios fundamentais da atenção à gestante, assinalados pela OMS, indicam que o cuidado na gestação deve ser multidisciplinar e multiprofissional, com atenção integral, levando em conta as necessidades intelectuais, emocionais, sociais e culturais das mulheres, filhos e famílias, e não somente um cuidado biológico. Deve também ser apropriado, tendo vista as diferentes pautas culturais para permitir lograr seus objetivos, compartilhar a tomada de decisão com as mulheres, respeitando a privacidade, dignidade e confidencialidade destas (BRASIL, 2005).

Várias estratégias vêm sendo criadas para que os princípios do Sistema Único de Saúde (SUS), como a Universalidade, Integralidade e a Equidade, possam ser operacionalizados pelos serviços de saúde. Neste sentido, surgem as Residências Multiprofissionais em Saúde com o desafio de remodelar a formação dos profissionais da área para o trabalho em equipe, na perspectiva da integralidade no cuidado, priorizando a saúde e não a doença (BRASIL, 2010, p. 260).

A Residência Multiprofissional em Saúde do Hospital Universitário Getúlio Vargas (HUGV), segundo a portaria interministerial n.506 de 24 de abril de 2008, constitui-se em uma pós-graduação latu sensu, oferecida às profissões que se relacionam com a saúde, caracterizada essencialmente por ensino em serviço. Possui dois programas articulados, voltados para a área de concentração Intensivismo e Saúde Funcional, que são: Atenção ao paciente Adulto Neurocirúrgico em UTI e Atenção Integral na Saúde Funcional em Doenças Neurológicas com atuação em hospitais e redes básicas de saúde dos Municípios e do Estado do Amazonas. A multiprofissionalidade nesses programas é articulada pelos profissionais Farmacêutico, Enfermeiro, Nutricionista, Psicólogo, Fisioterapeuta, Assistente Social e Educador Físico (HADDA, et al., 2006, p. 414).

No intuito de promover a experiência da integralidade da atenção à saúde nos vários níveis de sistema (primário, secundário e terciário), o projeto não fragmentou

LIMA, Janaina C. et al. Gestação Vida: oficina educacional para gestantes com abordagem multiprofissional em uma Unidade Básica de Saúde da cidade de Manaus. Extensão em Foco, Curitiba: Editora da UFPR, nr.10, jul/dez 2014, p.86-101. ISSN 2358-7180. 
a formação dos residentes, favorecendo o desenvolvimento com a articulação entre os serviços e ações de saúde, repercutindo efetivamente na qualidade de vida dos indivíduos.

A Equipe de Residência Multiprofissional do HUGV, através de sua atuação na Unidade Básica de Saúde (UBS), veio contribuir, a partir dessa prática inovadora, com a prevenção e promoção da saúde na comunidade do Armando Mendes através de um projeto de extensão, junto à Universidade Federal do Amazonas, que propunha ações educativas para as gestantes atendidas no pré-natal na UBS Geraldo Magela, na zona Leste de Manaus, através de uma oficina.

\section{Objetivo}

Este artigo tem como objetivo relatar a experiência de uma abordagem multiprofissional na educação em saúde, através de uma oficina para gestantes, sensibilizando-as quanto à importância de um pré-natal e de esclarecimento dos aspectos clínicos, psicológicos e sociais da gestação, bem como os cuidados necessários com os recém-nascidos.

\section{Captação para a realidade objetiva}

A atividade educativa foi desenvolvida na UBS Geraldo Magela, situada na zona Leste de Manaus(AM), durante as atividades práticas do primeiro ano da Residência Multiprofissional em Saúde, em junho de 2010.

Inicialmente foi realizada uma visita para reconhecimento e captação da realidade objetiva do bairro, da unidade de saúde, bem como da rotina estabelecida no local.

O Bairro Armando Mendes, caracterizado pela forma de ocupação urbana desordenada, que são as invasões, é uma área de grande expansão populacional onde predomina uma população de baixa renda e que enfrenta sérios problemas como prostituição, drogadição e violência. A UBS Geraldo Magela é a única Unidade de Saúde existente no bairro.

$\mathrm{Na}$ Unidade, após reconhecimento, verificou-se uma diversidade de programas de saúde e serviços implantados, como: Assistência Integral à Saúde da

LIMA, Janaina C. et al. Gestação Vida: oficina educacional para gestantes com abordagem multiprofissional em uma Unidade Básica de Saúde da cidade de Manaus. Extensão em Foco, Curitiba: Editora da UFPR, nr.10, jul/dez 2014, p.86-101. ISSN 2358-7180. 
Criança e da Mulher, Programa de Saúde Mental, Programa Nacional de Imunização, Programa de Combate à Malária, Saúde Bucal, Hipertensão Arterial e Diabetes, Controle das Doenças Sexualmente Transmissíveis, Programa de Combate à Hanseníase e Tuberculose, e alguns serviços como o de Enfermagem, Odontologia, Psicologia, Dermatologia e outros.

O perfil dos usuários que buscavam os serviços de saúde da UBS era caracterizado por um público jovem na idade produtiva, que possuíam baixa renda e baixa escolaridade. Os serviços que se destacaram por maior procura e demanda espontânea foi a consulta médica (clinico geral), consulta pediátrica, consulta de enfermagem para pré- natal e controle do Câncer de Colo de Útero e Mamas (PCCUM), consulta odontológica, serviço de enfermagem como curativo, inalação e vacina. Verificamos que a população buscava apenas os serviços médicos, tendo em vista que a unidade não oferecia outras atividades preventivas, com exceção do Programa de Saúde do Idoso.

O que também se pôde notar foi a pouca prática das atividades de Educação em Saúde voltadas ao coletivo, dando-se apenas prioridade, por diversos motivos, aos atendimentos individuais que, na maioria das vezes, eram realizados de maneira verticalizada, limitados ao espaço físico de uma sala de atendimento, com temas prontos, permitindo pouca ou nenhuma interação com a clientela.

\section{Interpretação da realidade objetiva}

Após reconhecimento das rotinas de atendimento e sua forma de funcionamento, surgiu o interesse da equipe multiprofissional em efetivar uma ação interdisciplinar dentro da unidade, aproveitando os programas nela implantados. Dentre as ações discorridas surge o esboço de alguns projetos, como o de atuação educativa para as grávidas atendidas na unidade, visto que, o atendimento periódico destas visava apenas ao acompanhamento do desenvolvimento do bebê, não tendo uma preocupação em fazer uma intervenção educativa para a futura mamãe. Somado a isto, deparávamos-nos com um agravante, a falta de ginecologista na unidade, aumentando com isso o interesse pela criação do projeto, visto que as práticas

LIMA, Janaina C. et al. Gestação Vida: oficina educacional para gestantes com abordagem multiprofissional em uma Unidade Básica de Saúde da cidade de Manaus. Extensão em Foco, Curitiba: Editora da UFPR, nr.10, jul/dez 2014, p.86-101. ISSN 2358-7180. 
educativas constituem parte indispensável das ações de saúde, em especial nas UBS, que em geral, desenvolvem diversos programas voltados para a saúde da população.

Rolim et al. (2006) afirma que esse déficit de oferta de atividades de Educação em Saúde, muitas vezes, é justificado pelos profissionais como sendo oriundo da insuficiente quantidade de equipes de saúde, do predomínio do modelo hospitalocêntrico, ausência ou inadequação de local para o desenvolvimento dessas atividades, disponibilização insuficiente de material de apoio e pela falta de qualificação e motivação desses profissionais.

Nessa direção, a educação em saúde não pode ocorrer como ação mecânica e automática, mas como uma ação planejada, dinâmica, sensível, múltipla e criativa, implicando vontade, ação, habilidades, técnica, ética, estética, que superam o conhecer, alegria, satisfação e prazer (SOUSA et al., 2007).

Baseado nisso e na grande demanda de atendimento do programa pré-natal, percebeu-se a necessidade de realizar uma estratégia de Educação em Saúde com foco multiprofissional e interdisciplinar em um grupo de gestantes, ressaltando a importância das mudanças físicas, psíquicas e sociais ocorridas durante esse período, através de uma oficina educativa, orientando-as a fim de supri-las de informações baseadas em cunho científico e não somente no saber popular, contribuindo para a saúde da gestante e do bebê.

Após o rastreamento através das fichas de cadastro do programa pré-natal, foi possível fazer um levantamento das grávidas que frequentavam a UBS e, diante disso, elaborar o planejamento da oficina, levando em conta a quantidade de grupos necessários e dias disponíveis para a abordagem. Durante os encontros para o planejamento, toda a equipe multiprofissional de residentes ficou encarregada de fazer o levantamento bibliográfico a respeito da temática, de elaborar sugestões e, posteriormente, de discutir todos os temas a serem abordados, tendo como base as recomendações do manual técnico do Ministério da Saúde (MS), "Pré-Natal e Puerpério: atenção qualificada e humanizada", bem como a realidade e necessidade das gestantes atendidas na UBS.

De posse dessas informações, seguiu-se a elaboração do projeto e divulgação da oficina, através de cartazes informativos com as datas do início e

LIMA, Janaina C. et al. Gestação Vida: oficina educacional para gestantes com abordagem multiprofissional em uma Unidade Básica de Saúde da cidade de Manaus. Extensão em Foco, Curitiba: Editora da UFPR, nr.10, jul/dez 2014, p.86-101. ISSN 2358-7180. 
término da oficina, as temáticas a serem abordadas e a descrição do público alvo. Os impressos foram fixados na unidade local, no Centro de Convivência do Idoso (CCl) anexado a UBS, na Unidade Básica de Saúde da Família (UBSF) e na Associação dos moradores do bairro, juntamente com a colaboração dos próprios funcionários. Posteriormente, deu-se o início das oficinas como exemplificado no mapa conceitual elaborado no planejamento (Figura 1).

\section{FIGURA 1 - DIAGNÓSTICO SITUACIONAL}

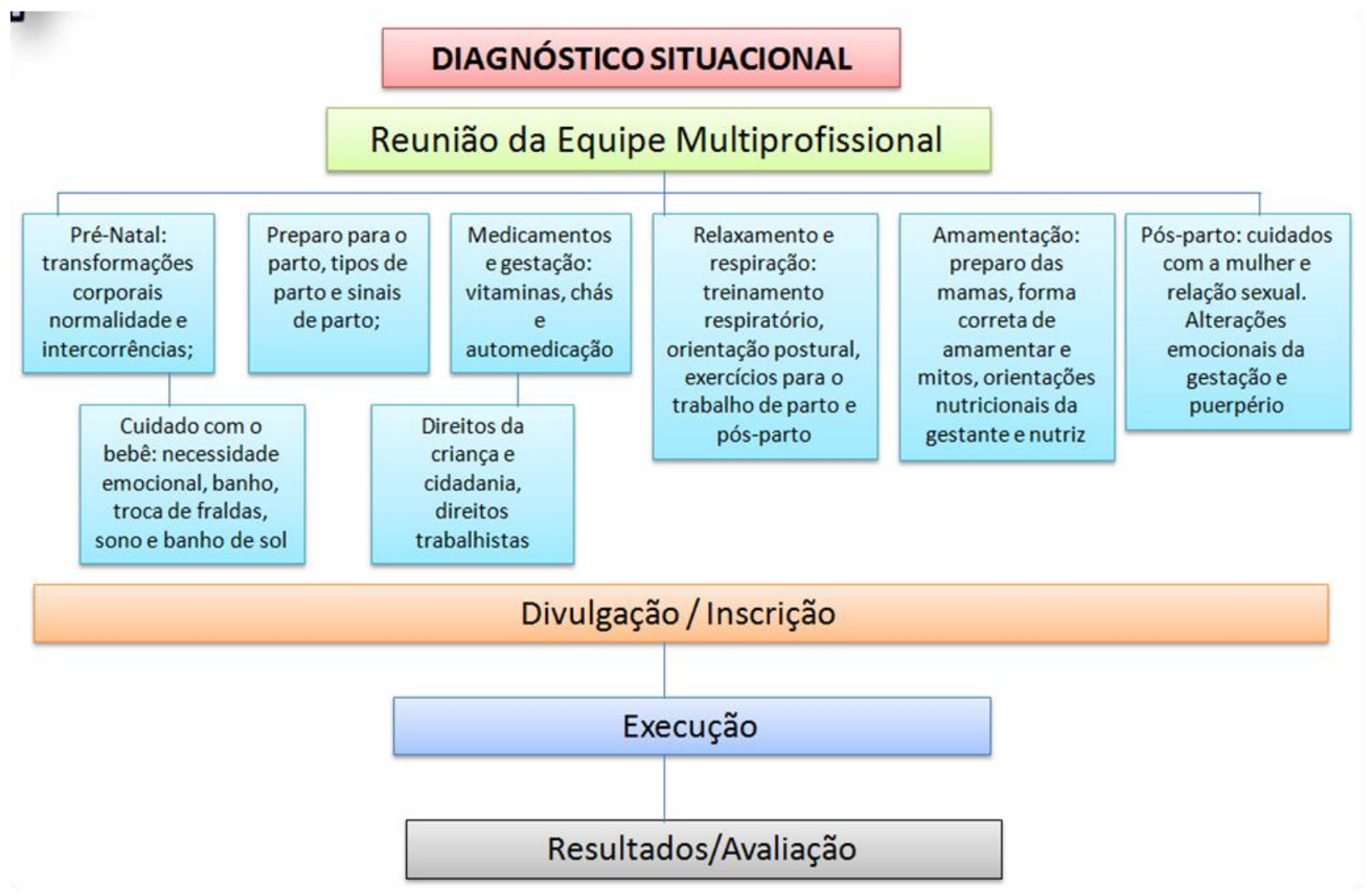

FONTE: Arquivo Pessoal. Mapa Conceitual do Planejamento Estratégico.

A procura para realização das inscrições pelas grávidas se davam por livre e espontânea vontade, sendo entregue no momento da inscrição um calendário informativo com os dias de cada encontro e principais temas a serem abordados na oficina.

LIMA, Janaina C. et al. Gestação Vida: oficina educacional para gestantes com abordagem multiprofissional em uma Unidade Básica de Saúde da cidade de Manaus. Extensão em Foco, Curitiba: Editora da UFPR, nr.10, jul/dez 2014, p.86-101. ISSN 2358-7180. 


\section{Construção do projeto de intervenção na realidade objetiva}

A priori, procurou-se estabelecer com as gestantes uma relação empática, por meio de conversas informais, visando à aproximação para manter uma relação de confiança onde houvesse a prática da escuta ativa por parte dos profissionais de saúde envolvidos. Escolheu-se, portanto, uma abordagem em grupo, onde as cadeiras eram dispostas em semicírculo, de forma que houvesse interação e troca de informação entre elas (Figura 2). A estratégia usada pela equipe Multiprofissional foi a realização de diálogos expositivos e atividades práticas, como as atividades físicas, alongamentos, exercícios respiratórios e posturais e também técnicas de relaxamento. Para os diálogos expositivos, fez-se uso de recurso audiovisual como data show, entrega de folhetos explicativos personalizados ou cedidos pela própria unidade, bem como demonstrações práticas, videoaulas e sorteios de brindes.

\section{FIGURA 2 - DISPOSIÇÃO DO GRUPO}

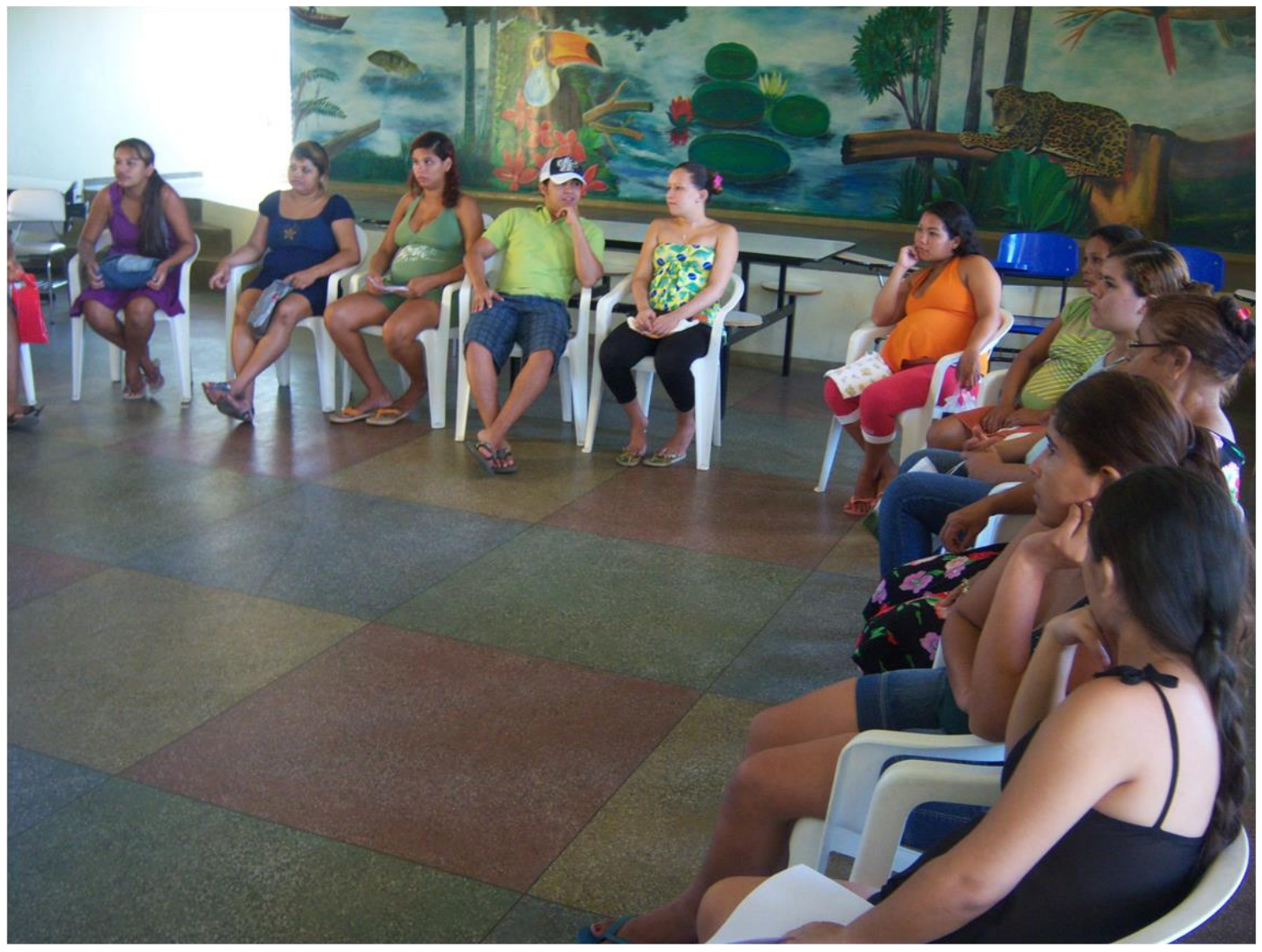

FONTE: Arquivo pessoal. Disposição das cadeiras permitindo interação entre as participantes.

LIMA, Janaina C. et al. Gestação Vida: oficina educacional para gestantes com abordagem multiprofissional em uma Unidade Básica de Saúde da cidade de Manaus. Extensão em Foco, Curitiba: Editora da UFPR, nr.10, jul/dez 2014, p.86-101. ISSN 2358-7180. 
As atividades educativas foram ministradas em um espaço cedido pelo Centro de Convivência do Idoso (CCI) que é anexado a UBS. Foram acompanhadas duas turmas, a duração de cada oficina consistiu em aproximadamente dois meses, apenas uma vez na semana (às segundas-feiras), com duração de duas horas cada módulo, sendo reservada uma hora para a abordagem do tema, trinta minutos para atividades práticas, e nos trinta minutos finais eram realizados sorteios de brindes e socialização.

A oficina apresentou módulos com as seguintes temáticas:

- Pré-Natal: transformações corporais, normalidade e intercorrências;

- Preparo para o parto, tipos de parto e sinais de parto;

- Medicamentos e gestação: vitaminas, chás e automedicação;

- Cuidado com o bebê: necessidade emocional, banho, troca de fraldas, sono e banho de sol;

- Direitos da criança e cidadania, direitos trabalhistas;

- Amamentação: preparo das mamas, forma correta de amamentar e mitos, orientações nutricionais da gestante e nutriz;

- Relaxamento e respiração: treinamento respiratório, orientação postural, exercícios para o trabalho de parto e pós-parto;

- Pós-parto: cuidados com a mulher e relação sexual. Alterações emocionais da gestação e puerpério.

Estas estratégias foram elaboradas para que as atividades ocorressem de forma dinâmica e envolvente, onde se oportunizou a participação de todos, estreitando o elo entre profissional e clientela, com o intuito de alcançar o objetivo proposto.

\section{Intervenção na realidade objetiva}

Para Hoga e Reberte (2007), é essencial a existência de um serviço com atendimento interdisciplinar que aborde tanto os aspectos psicológicos quanto físicos, visando a um atendimento holístico à gestante. Diante disso, a equipe multiprofissional valorizou uma abordagem interdisciplinar, onde cada profissional pôde trazer contribuições de diversas áreas de conhecimento, favorecendo assim, a educação de forma integral.

LIMA, Janaina C. et al. Gestação Vida: oficina educacional para gestantes com abordagem multiprofissional em uma Unidade Básica de Saúde da cidade de Manaus. Extensão em Foco, Curitiba: Editora da UFPR, nr.10, jul/dez 2014, p.86-101. ISSN 2358-7180. 
A abordagem pelo profissional farmacêutico visou priorizar a importância da adesão à suplementação vitamínica, bem como o uso do ferro, colocando em grande destaque a problemática da automedicação e uso indiscriminado dos chás. Visto que o uso regular de medicamentos é frequente entre as gestantes, e estima-se que $90 \%$ das mulheres consumam pelo menos um medicamento durante a gestação. Dentre esses, existem substâncias consideradas seguras ou sem evidência de risco e que, se necessário, devem ser bem indicados e utilizados (BRASIL, 2011, p. 240).

As orientações relacionadas com a amamentação e a sua importância durante a gestação foram abordadas de forma especial, utilizando videoaula para a explanação, bem como aula prática utilizando como modelo mães presentes que ainda amamentavam seus filhos (Figura 3), possibilitando uma maior associação e compreensão, contribuindo desta forma para a adesão e continuidade do aleitamento materno ao longo do desenvolvimento do bebê.

\section{FIGURA 3 - EXPOSIÇÕES PRÁTICAS}

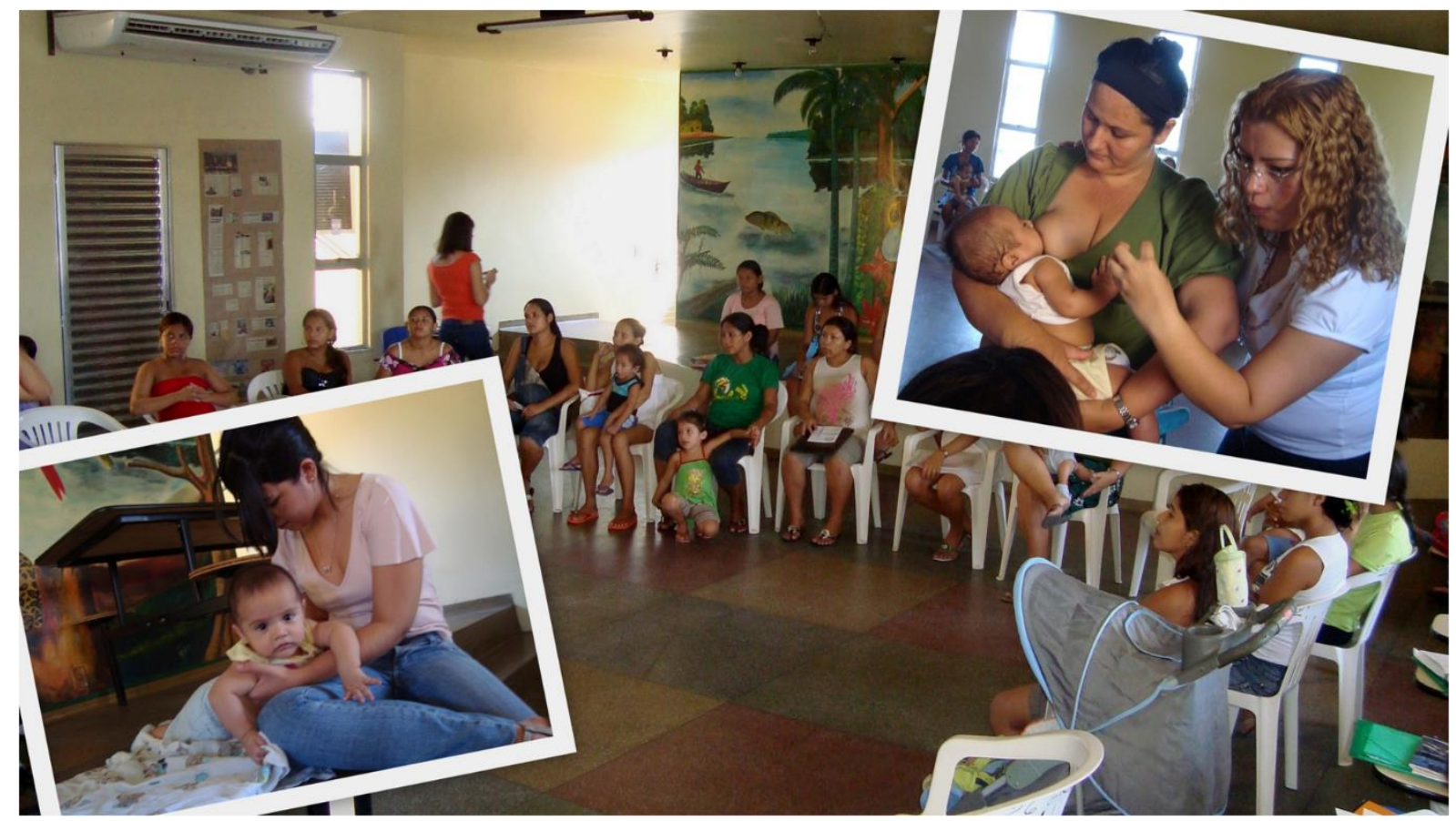

FONTE: Arquivo pessoal. Exposição prática do tema amamentação e cuidados com o recémnascido.

LIMA, Janaina C. et al. Gestação Vida: oficina educacional para gestantes com abordagem multiprofissional em uma Unidade Básica de Saúde da cidade de Manaus. Extensão em Foco, Curitiba: Editora da UFPR, nr.10, jul/dez 2014, p.86-101. ISSN 2358-7180. 
O profissional nutricionista visou à explanação da importância de uma boa alimentação e a desmistificação de alguns hábitos alimentares, tendo em vista a necessidade de uma alimentação equilibrada em nutrientes tanto para si quanto para seu bebê. Houve a necessidade de propor novas alternativas através da valorização dos produtos regionais da Amazônia e também da importância do reaproveitamento alimentar, adequando a realidade ao contexto de vida das gestantes carentes.

Brasil (2011) diz que o cuidado nutricional no pré-natal tem o seu valor pelo impacto no resultado obstétrico tornando-se de grande relevância para a saúde pública.

Devido às dificuldades enfrentadas em se conseguir materiais expositivos para utilizar nas abordagens práticas, como bonecos, banheiras, entre outros, o profissional enfermeiro também optou pela utilização de vídeos educativos e autoexplicativos para os temas que exigiam uma demonstração mais prática, tais como: troca de fraldas, banho no recém-nascido e higienização do coto umbilical.

Os vídeos adquiridos pelo sistema de busca online oportunizou transmitir uma mensagem baseada em um conteúdo seguro, visto que a equipe realizou a prévia seleção e aprovação desses. Pelo fato de serem atrativos, os vídeos despertaram e fixaram a atenção das participantes e, mesmo diante dessa abordagem, priorizou-se estabelecer uma participação mais ativa, onde as experiências trazidas pelas participantes fizessem parte da discussão.

Falcone et al (2005) citam que a gravidez pode ser considerada como um fator gerador de ansiedade dentre outras causas, tornando a mulher mais vulnerável ao desenvolvimento das perturbações emocionais.

A disponibilidade para ouvir a gestante com uma postura de acolhimento é o requisito mais importante para a ação preventiva. Por meio dessa interação, cada profissional pode detectar as mudanças de comportamento, variações de humor e de pensamento. A abordagem pelo profissional psicólogo com as gestantes possibilitou momentos onde elas pudessem expor sem receio seus sentimentos e medos ao grupo.

A legislação brasileira prevê a proteção dos direitos das mulheres e das gestantes, inclusive na seção referente aos direitos dos trabalhadores, enfatizando a proteção da mulher no mercado de trabalho e na sociedade, mediante dispositivos

LIMA, Janaina C. et al. Gestação Vida: oficina educacional para gestantes com abordagem multiprofissional em uma Unidade Básica de Saúde da cidade de Manaus. Extensão em Foco, Curitiba: Editora da UFPR, nr.10, jul/dez 2014, p.86-101. ISSN 2358-7180. 
específicos. Ao abordar os direitos sociais relacionados à gestação, a Assistente Social pode colocar questões de comum interesse entre elas, como os direitos trabalhistas e sociais da mulher.

Através da atuação da Educadora Física, as gestantes foram incentivadas à manutenção de uma atividade física habitual, proporcionando a elas um momento de ginástica orientada, evitando assim a prática indiscriminada de exercícios errôneos e prejudiciais à saúde. Com a integração do Fisioterapeuta, atividades voltadas para a correção postural, exercícios respiratórios, exercícios para fortalecimento do assoalho pélvico e técnicas de relaxamento foram aplicadas (Figura 4).

O relaxamento e a massagem propiciam à gestante superar suas ansiedades ou minimizá-las, para que consiga encontrar o melhor caminho para viver a gestação com mais equilíbrio e ter um parto tranquilo (FALCONE, et al. 2005, p. 612-618).

\section{FIGURA 4 - ATIVIDADE FÍSICA}

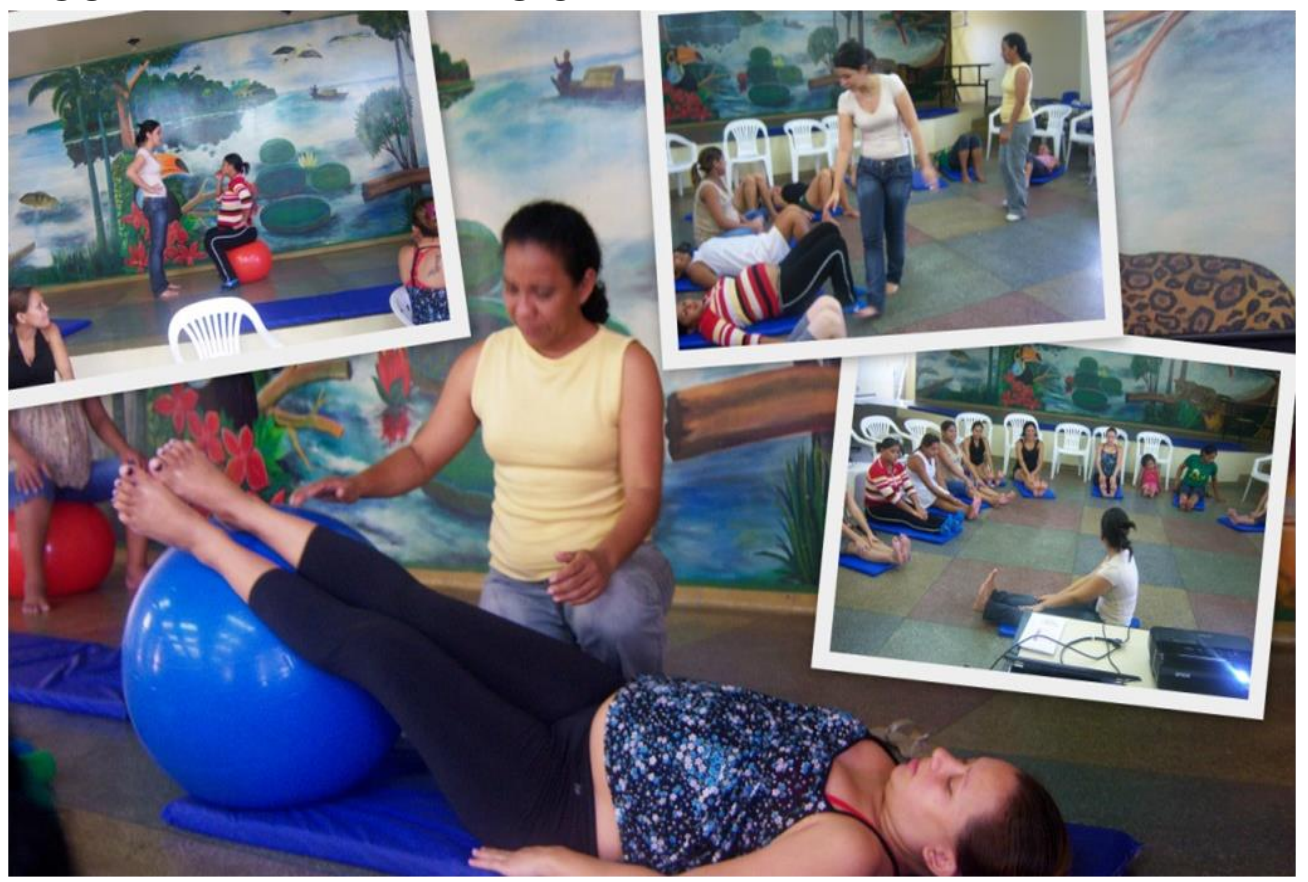

FONTE: Arquivo pessoal. Atividades físicas e fisioterápicas.

Para ampliar o conhecimento e favorecer a continuidade do aprendizado da gestante no seu lar com a sua família, foram oportunizados folhetos explicativos

LIMA, Janaina C. et al. Gestação Vida: oficina educacional para gestantes com abordagem multiprofissional em uma Unidade Básica de Saúde da cidade de Manaus. Extensão em Foco, Curitiba: Editora da UFPR, nr.10, jul/dez 2014, p.86-101. ISSN 2358-7180. 
personalizados (Figura 5), com valorização visual, para atender a todas as necessidades das presentes, como no caso, uma gestante deficiente auditiva.

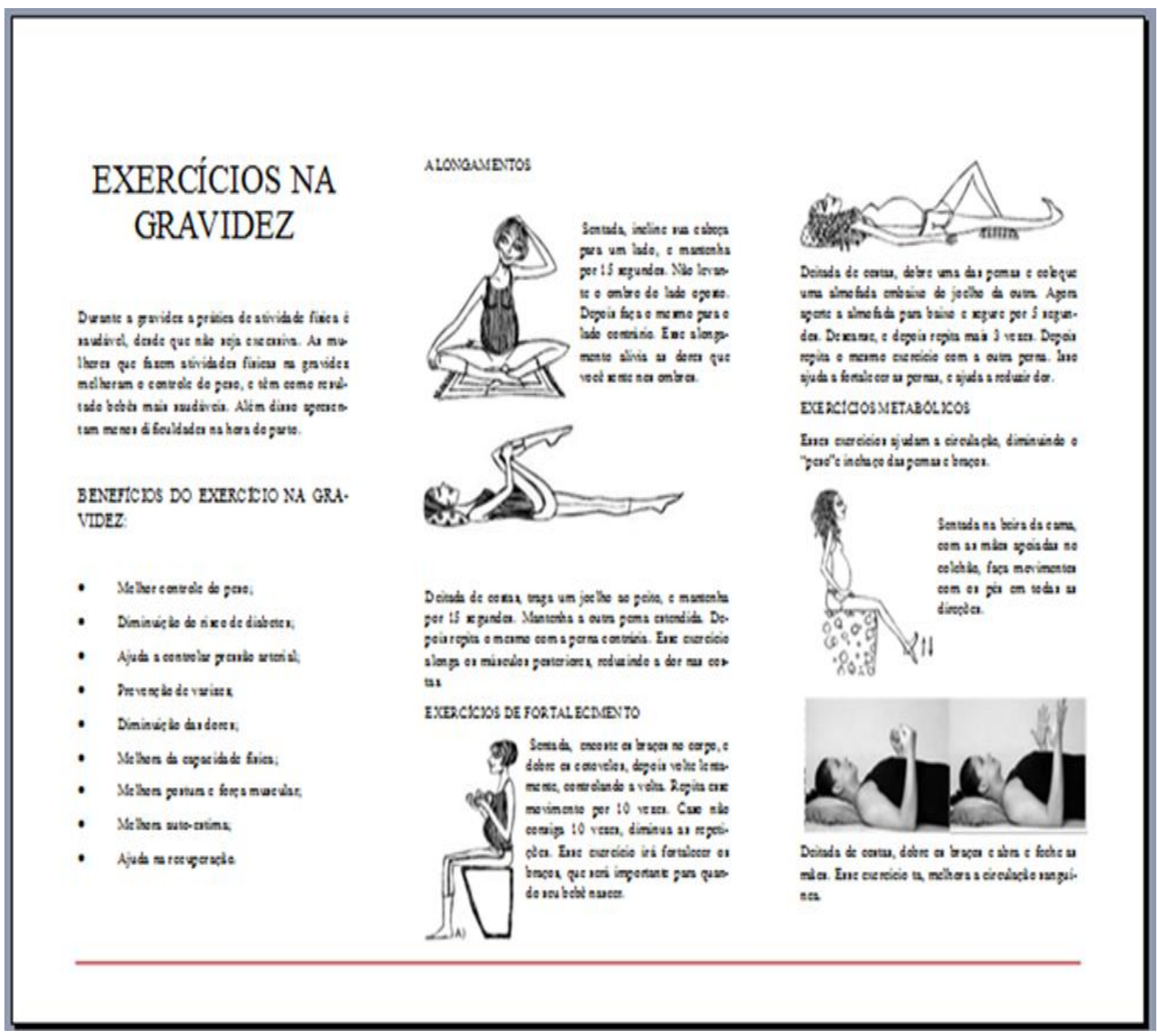

FONTE: Arquivo pessoal. Folhetos explicativos personalizados para a abordagem de exercícios físicos na gravidez.

As dinâmicas de aproximação utilizadas na realização dos encontros possibilitaram maior integração entre as gestantes, facilitando o conhecimento e reconhecimento entre elas, suas características comuns e suas peculiaridades. Além disso, possibilitaram a participação delas nas discussões dos assuntos propostos, de

LIMA, Janaina C. et al. Gestação Vida: oficina educacional para gestantes com abordagem multiprofissional em uma Unidade Básica de Saúde da cidade de Manaus. Extensão em Foco, Curitiba: Editora da UFPR, nr.10, jul/dez 2014, p.86-101. ISSN 2358-7180. 
maneira que se sentissem à vontade para questionar, opinar, sugerir e discordar. Enfim, criou-se um espaço de troca e compartilhamento de saberes e experiências.

Através das anotações no diário de campo e com a observação das participantes, constatamos que durante os encontros dos grupos educativos, de forma geral, as gestantes demonstraram-se tímidas. No entanto, com a evolução dos encontros, foram demonstrando mais confiança, mais segurança no ambiente e nas pessoas presentes que também vivenciavam as mesmas emoções, transformações, medos e inseguranças.

Outro fator relevante presenciado foi o vínculo de confiança estabelecido entre elas e a equipe multiprofissional, mesmo que algumas das gestantes, por timidez, não tenham se manifestado durante a oficina, pois ao final dos encontros as nossas atenções foram solicitadas para questionar ou tirar dúvidas a respeito da sua gestação. Isto demonstra que elas estabeleceram vínculo de confiança com a equipe, fruto dos vários encontros, conversas e trocas.

\section{Considerações finais}

A experiência como Residentes na atenção básica nos mostrou a possibilidade de repensar sobre a nossa prática profissional atual, baseada apenas na clínica tradicional, nos remetendo para além do individual através de um modelo assistencial fundamentado na multidisciplinaridade, integralidade e na humanização do cuidado, demonstrando que é possível a ampliação do trabalho voltado para a complexidade do processo gravídico e puerperal.

A oficina repercutiu de forma positiva entre as participantes, pois suas dúvidas foram respondidas e estas afirmaram estar mais tranquilas e seguras apesar de muitas não estarem na sua primeira gestação. Todos os resultados alcançados demonstraram plenamente, sob diferentes olhares e pontos de vista, que a realização desta oficina se revelou de grande valia, visto que percebemos uma carência de assistência Multiprofissional e Interprofissional às gestantes, que nesta fase da vida, encontram-se necessitadas de informações e apoios. Não foi planejada a utilização de questionários qualitativos para avaliação, mas obtivemos o retorno baseado na oralidade a partir das trocas de experiências no grupo.

LIMA, Janaina C. et al. Gestação Vida: oficina educacional para gestantes com abordagem multiprofissional em uma Unidade Básica de Saúde da cidade de Manaus. Extensão em Foco, Curitiba: Editora da UFPR, nr.10, jul/dez 2014, p.86-101. ISSN 2358-7180. 
FIGURA 6 - PRIMEIRA TURMA DA OFICINA

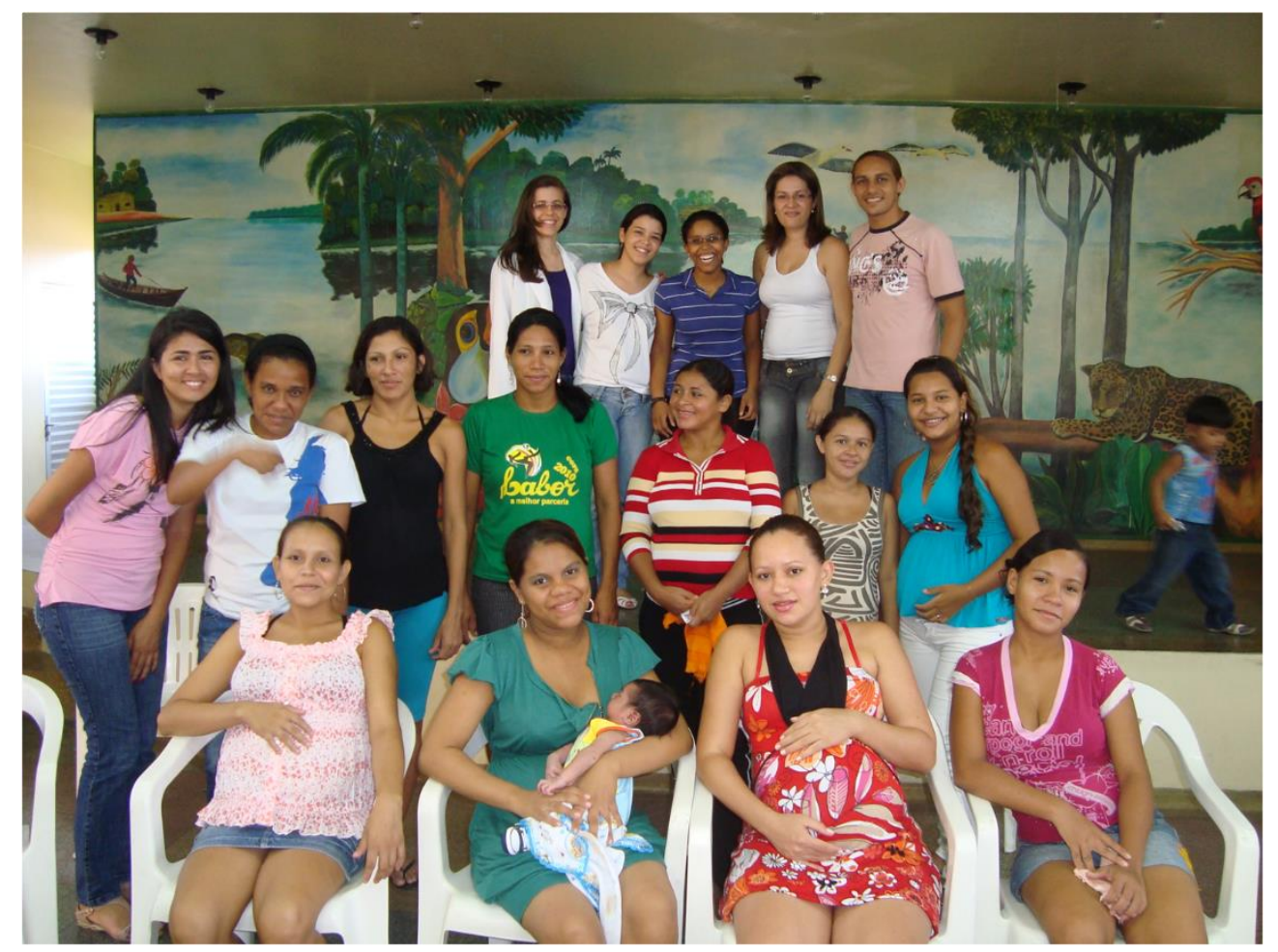

FONTE: Arquivo pessoal. Grávidas da primeira oficina e equipe Multiprofissional.

As trocas de conhecimento adquiridas foram muito relevantes, já que acreditamos que os objetivos propostos foram atingidos, pois verificou-se que foi possível conhecer melhor as gestantes, bem como seus anseios e perspectivas relacionadas à gestação.

Isto acaba por evidenciar a importância de outras instituições de saúde, tanto públicas como privadas, em investirem em Educação em Saúde. Essas ações merecem continuidade para que causem impacto na qualidade de vida das gestantes e possam contribuir para a qualificação do SUS. Sugere-se, portanto, que haja comprometimento entre os profissionais, gestores e comunidade na implementação de ações educativas com enfoque na promoção da saúde para a melhoria da qualidade de vida no pré-natal.

LIMA, Janaina C. et al. Gestação Vida: oficina educacional para gestantes com abordagem multiprofissional em uma Unidade Básica de Saúde da cidade de Manaus. Extensão em Foco, Curitiba: Editora da UFPR, nr.10, jul/dez 2014, p.86-101. ISSN 2358-7180. 


\section{Referências}

BRASIL. Ministério da Saúde. Grupo Hospitalar Conceição. Gerência de Saúde Comunitária. Atenção à saúde da gestante em APS. Organização de Maria Lucia Medeiros Lenz e Rui Flores. Porto Alegre: Hospital Nossa Senhora da Conceição, 2011. p. 240.

BRASIL. Ministério da Saúde. Secretaria de Atenção à Saúde. Departamento de ações Programáticas Estratégicas. Área Técnica de Saúde da Mulher. Pré-natal e Puerpério: atenção qualificada e humanizada - manual técnico. Ministério da Saúde, 2005.

BRASIL. Ministério da Saúde. Grupo Hospitalar Conceição. Residências em saúde: fazeres \& saberes na formação em saúde. Organização de Ananyr Porto Fajardo, Cristianne Maria Famer Rocha e Vera Lúcia Pasini. Porto Alegre: Hospital Nossa Senhora da Conceição, 2010. p. 260.

BRASIL. Ministério da Educação - MEC. Portaria interministerial n. 506, de 24 de abril de 2008.

CAMACHO, K. G. et al. Vivenciando repercussões e transformações de uma gestação: perspectivas de gestantes. Ciencia y Enfermeria, v. 16 n. 2, p. 115-125, 2010.

FALCONE, V. M. et al. Atuação multiprofissional e a saúde mental de gestantes. Revista de Saúde Pública, v. 39, n. 4, p. 612-618, 2005.

HADDAD, A. E. et al. Residência Multiprofissional em Saúde: experiências, avanços e desafios. Ministério da Saúde, 2006. p. 414.

HOGA, L. A. K.; REBERTE, L. M. Pesquisa ação como estratégia para desenvolver grupo de gestantes: a percepção dos participantes. Rev. Esc. Enferm. USP, v. 4, n. 4, p. 559-566, dez. 2007.

REIS, D. M. et al. Educação em saúde como estratégia de promoção de saúde bucal em gestantes. Ciênc. \& Saúde Coletiva, Rio de Janeiro, v. 15, n. 1, p. 269-276, jan. 2010.

ROLIM, M. O. et al. Curso para gestantes: ação educativa na perspectiva da coresponsabilidade. Online Brazilian Journal of Nursing, v. 5, n. 3, 2006.

SOUZA, F. G. M. et al. Educação em Saúde, enfermeiros e criatividade: a interconexão necessária para o processo educativo. Online Brazilian Journal of Nursing, v. 6, n. 2, 2007. Disponível em: <http://www.uff.br/objnursing/index.php/ nursing/rt/printerFriendly/j.1676-4285.2007>. Acesso em: 19/03/2012.

LIMA, Janaina C. et al. Gestação Vida: oficina educacional para gestantes com abordagem multiprofissional em uma Unidade Básica de Saúde da cidade de Manaus. Extensão em Foco, Curitiba: Editora da UFPR, nr.10, jul/dez 2014, p.86-101. ISSN 2358-7180. 